\title{
In vitro assessment of iron availability from commercial Young Child Formulae supplemented with prebiotics
}

\author{
Tatiana Christides $^{1}$ (i) $\cdot$ Julia Clark Ganis $^{1} \cdot$ Paul Anthony Sharp $^{2}$
}

Received: 9 February 2016 / Accepted: 29 November 2016 / Published online: 9 December 2016

(C) The Author(s) 2016. This article is published with open access at Springerlink.com

\begin{abstract}
Purpose Iron is essential for development and growth in young children; unfortunately, iron deficiency (ID) is a significant public health problem in this population. Young Child Formulae (YCF), milk-derived products fortified with iron and ascorbic acid (AA, an enhancer of iron absorption) may be good sources of iron to help prevent ID. Furthermore, some YCF are supplemented with prebiotics, non-digestible carbohydrates suggested to enhance iron bioavailability. The aim of our study was to evaluate iron bioavailability of YCF relative to prebiotic and AA concentrations. We hypothesised that YCF with the highest levels of prebiotics and AA would have the most bioavailable iron.

Methods We used the in vitro digestion/Caco-2 cell model to measure iron bioavailability from 4 commercially available YCF with approximately equal amounts of iron, but varying amounts of: AA and the prebiotics fructo- and galacto-oligosaccharides. Caco-2 cell ferritin formation was used as a surrogate marker for iron bioavailability.

Results The YCF with the highest concentration of prebiotics and AA had the highest iron bioavailability; conversely, the YCF with the lowest concentration of prebiotics and AA had the lowest. After the addition of exogenous prebiotics, so that all tested YCF had equivalent amounts, there
\end{abstract}

Tatiana Christides

T.Christides@gre.ac.uk

1 Department of Life and Sports Sciences, Faculty of Engineering and Science, University of Greenwich, Medway Campus, Chatham Maritime, Kent ME4 4TB, UK

2 Metal Metabolism Group, Diabetes and Nutritional Sciences Division, School of Medicine, King's College London, London, UK was no longer a significant difference between YCF iron bioavailability.

Conclusion Our results suggest that ascorbic acid and prebiotics in YCF improve iron bioavailability. Ensuring that iron is delivered in a bioavailable form would improve the nutritional benefits of YCF in relation to ID/IDA amongst young children; therefore, further exploration of our findings in vivo is warranted.

Keywords Iron · Bioavailability $\cdot$ Prebiotics $\cdot$ Young children · Young Child Formulae $\cdot$ Caco-2

\section{Introduction}

Iron is essential for growth and neurodevelopment in infants and young children (children between the ages of 1-3). Humans from birth until 3 years of age undergo rapid brain growth and development; iron is required for multiple processes underlying this developmental surge including myelination, neurotransmitter function, brain energy homoeostasis, and neuronal and synaptogenesis [1]. Inadequate iron during this period of childhood is associated with impaired cognitive and psychomotor development that may not reverse even if children become iron-replete later in life [2]. Full-term infants are protected from iron deficiency and iron deficiency anaemia (ID/IDA) for the first 4-6 months after birth as a result of gestationally derived iron stores [3]. Unfortunately, for the rest of the critical time period up to 3 years of age, children are at high risk for ID/IDA because of rapid overall growth and associated increased iron needs and often inadequate intake of bioavailable dietary iron $[3,4]$.

Infant formula is fortified with iron to prevent deficiency [5], and the evidence suggests that this mandatory 
fortification is effective-in the developed world less than $5 \%$ of infants under 12 months have IDA [3, 6]. However, the prevalence of ID/IDA increases between 1-3 years of age, ranging from 6 to $15 \%$ in the developed world [7, 8], due to accelerated growth at this developmental stage, and also possibly the switch from formula to cow's milk that has less iron compared with formula [9-11]. In response, the food industry has developed "Young Child Formulae" (YCF), milk-derived products fortified with multiple micronutrients and other dietary factors, aimed at 1- to 3-year-old children. YCF contain more iron compared with cow's milk and are also fortified with ascorbic acid, a known enhancer of non-haem iron absorption.

There are numerous commercially available YCF throughout the developed world. In the United Kingdom (UK), according to the Diet and Nutrition Survey of Infants and Young children, 18\% of children between 12 and 18 months received YCF as part of their diet, representing a significant number of children consuming these products [12]. Furthermore, a cross-sectional study of 1- to 2-yearold children conducted in France found that use of YCF was associated with improved dietary iron intake compared with children exclusively receiving cow's milk [10]; however, the specific nutrient composition of the YCF being consumed with reference to modifiers of iron absorption was not specified, nor were iron status markers measured in study subjects to assess whether the observed increase in iron intake correlated with good iron status.

In addition to added iron and ascorbic acid, several YCF products are supplemented with prebiotics. Prebiotics are non-digestible dietary substances, typically oligosaccharides such as fructo- and galacto-oligosaccharides (FOS and GOS, respectively) that encourage the growth of beneficial microorganisms in the large intestine [13, 14]. Prebiotics have also been shown to improve the bioavailability of minerals, although results with regard to iron are not consistent [15]. The type and amount of added prebiotic varies between YCF, and some YCF do not have any added prebiotics (source of information: manufacturers' nutrition labels and websites). Of note, The European Society for Pediatric Gastroenterology, Hepatology and Nutrition (ESPGHAN) Committee on Nutrition in a recent review found that there was no evidence of adverse effects from the addition of prebiotics to formula; however, they also concluded that evidence was insufficient to recommend routine supplementation and that further research was needed [16]. The European Commission regulates infant and follow-on formulae composition in the European Union and stipulates a maximum amount of prebiotics that may be added, but does not make more specific reference to recommended amounts (Commission Directive 2006/141/ EC, reviewed and updated July 2016) [17].
Although young children are at increased risk for ID/ IDA, there is also concern that excess dietary iron may affect their gut microbiome with negative local and systemic health effects [18]. A study conducted in Kenya found that intake of iron-fortified foods led to a higher number of gut pathogens, increased intestinal inflammation and more frequent diarrhoea episodes requiring treatment [19]. In another study, consumption of iron-fortified biscuits was associated with an unfavourably altered gut microbiome [20]. Unabsorbed iron reaching the colon is suggested to mediate these effects [21]. Of note, however, a study in children from 9 to 18 months living in the UK, who continued to receive iron supplemented formula, found no evidence of adverse effects over the 9 months of the study even in iron-replete children [22]; thus, the effects of iron on the young gut may vary depending on underlying risk for gut inflammation and infection. Nonetheless, foods fortified with highly bioavailable iron are desirable because of their efficacy in improving iron nutriture as a result of increased absorption. They may also, as a consequence of improved small bowel uptake, deliver less iron to the large intestine that may have long-term health implications related to the gut microbiome.

The objective of this study was to compare the iron bioavailability of four YCF commonly consumed in the UK containing approximately equal amounts of iron, but with different ascorbic acid concentrations, and varying amounts and types of FOS and GOS. The aim of our research was to evaluate YCF iron bioavailability relative to supplemental prebiotic and ascorbic acid concentrations. We utilised the Caco-2 cell/in vitro digestion model with the formation of Caco-2 cell ferritin as a surrogate marker for cellular iron uptake. This model has been used in multiple studies to assess iron bioavailability from foodstuffs including milks, milk-derived products and infant formulae, for examples see [23-25]. We hypothesised that the YCF with the highest concentrations of prebiotics and ascorbic acid would have the highest levels of bioavailable iron, while the YCF with no added prebiotics and lower levels of ascorbic acid would have the lowest iron availability.

\section{Materials and methods}

\section{Reagents}

All chemicals and enzymes, unless otherwise stated, were purchased from Sigma-Aldrich (UK). Acids required for glassware cleaning and in vitro digestions were acquired from VWR (UK). Thermo Fisher Scientific was the provider of cell culture media, flasks, tissue culture plates and cell culture reagents. Twenty-four hours prior to each 
Table 1 Nutrient composition of YCFs A-D according to the manufacturers' labels and websites

\begin{tabular}{lllll}
\hline Nutrient $(\mathrm{mg} / 100 \mathrm{ml}$ unless otherwise noted $)$ & \multicolumn{2}{l}{ YCF sample } & $\mathrm{C}$ & $\mathrm{D}$ \\
\cline { 2 - 5 } & $\mathrm{A}$ & $\mathrm{B}$ & 10 & 12 \\
\hline Ascorbic acid & 15 & 15 & 1.1 & 1.2 \\
Iron & 1.2 & 1.2 & $\left(\mathrm{FeSO}_{4}\right)$ & $\left(\mathrm{FeSO}_{4}\right)$ \\
& $\left(\mathrm{FeSO}_{4}\right)$ & $\left(\mathrm{Fe}_{2}\right.$ lactate $)$ & 0 & 0 \\
FOS & 120 & Not stated & 0 & 0 \\
GOS & 1080 & Not stated & 500 & 0 \\
Total FOS and GOS & 1200 & 1200 & $\mathrm{~N} / \mathrm{a}$ & 0 \\
Fibre $(\mathrm{g} / 100 \mathrm{ml})$ & 0.8 & 0.8 & 0.5 & 0 \\
\hline
\end{tabular}

experiment glass- and plastic-ware needed for experiments were soaked in $10 \%(\mathrm{v} / \mathrm{v})$ trace metal grade $68 \%$ nitric acid and then subsequently rinsed with $18 \mathrm{~m} \Omega$ pure water.

GOS powders (100\% purity) used in experiments were either purchased from Megazyme International (Ireland), or kindly provided by King-Prebiotics. FOS powder (100\% purity) was purchased from Health Plus (Seaford, East Sussex).

\section{Samples}

Four YCF were obtained from three different leading UK supermarkets on three separate occasions, herein identified as YCF A, B, C and D. Products were tested before the use by date and within 1 month of purchase. YCF were stored unopened at room temperature, similar to their distribution and market environment. YCF A and B were fortified with equivalent amounts of prebiotics in the forms of GOS and FOS by the manufacturer; YCF A specified the ratio of GOS to FOS, but neither YCF A nor B provided nutrient label information on the specific forms of GOS and FOS used. YCF C was fortified with GOS alone by the manufacturer, and at a lower concentration compared with YCF A and B, and YCF D had no added prebiotics. YCF A, B and D had $1.2 \mathrm{mg}$ iron $/ 100 \mathrm{ml}$ product; YCF $\mathrm{C}$ had $1.1 \mathrm{mg} / 100 \mathrm{ml}$. Ascorbic acid levels also differed between YCF; YCF A and B had the highest reported levels, followed by YCF D. YCF C had the lowest reported concentration of vitamin $\mathrm{C}$. The nutrient composition of the various YCF in relation to total iron, ascorbic acid and prebiotics is provided below (Table 1).

\section{Cell culture}

The TC7 Caco- 2 cell line was used for all experiments (kindly endowed to the Sharp laboratory by Monique Rousset and Edith Brot-Laroche, INSERM U505, [26]); work with this cell line investigating iron uptake and bioavailability has been published in numerous studies, for example [27-29].
Details of the in vitro digestion method have been previously published $[27,28,30]$. Briefly, cells were grown in T75 flasks and seeded into six-well tissue culture plates for experiments. According to the method developed by the Glahn laboratory [30], the in vitro digestions were carried out 13-15 days post-seeding. The media used to feed cells was Dulbecco's Modified Eagle Medium supplemented with $10 \% \mathrm{v} / \mathrm{v}$ foetal bovine serum, $1 \%$ penicillin-streptomycin, $4 \mathrm{mmol} / \mathrm{L}$ L-glutamine, $1 \%$ non-essential amino acids.

Media were changed to supplemented MEM $(10 \mathrm{mmol} / \mathrm{L}$ PIPES [piperazine- $\mathrm{N}, \mathrm{N}^{\prime}$-bis (2-ethanesulfonic acid)], $1 \%$ antibiotic/antimycotic solution, $11 \mu \mathrm{mol} / \mathrm{L}$ hydrocortisone, $0.87 \mu \mathrm{mol} / \mathrm{L}$ insulin, $0.02 \mu \mathrm{mol} / \mathrm{L}$ sodium selenite $\left(\mathrm{Na}_{2} \mathrm{SeO}_{3}\right), 0.05 \mu \mathrm{mol} / \mathrm{L}$ triiodothyronine and $20 \mu \mathrm{g} / \mathrm{L}$ epidermal growth factor), without foetal bovine serum, to ensure satisfactory cell growth but with low basal media iron levels [30, 31].

\section{YCF and in vitro digestions}

All experiments were carried out with freshly prepared reagents and freshly opened cartons of YCF; only liquid forms of YCF were tested in experiments. 1.8-2 ml of YCF sample was used per digestate depending on sample iron concentration.

All experiments contained a set of controls: a digest with no added iron to ensure no iron contamination of our system (Blank food digest); a reference digest of $30 \mu \mathrm{mol} / \mathrm{L}$ $\mathrm{Fe}$ ( $\mathrm{Fe}$ alone) equivalent to the iron concentration in $\mathrm{YCF}$ digestates; and a positive control digest of $30 \mu \mathrm{mol} / \mathrm{L} \mathrm{Fe}$ and $300 \mu \mathrm{mol} / \mathrm{L}$ ascorbic acid (Fe + AA at a 1:10 molar ratio).

The digests were prepared as previously described [8]. Briefly, pepsin was added to the controls or YCF samples to initiate the gastric phase of the in vitro digestion (tested controls or samples herein referred to as digestates), and the $\mathrm{pH}$ adjusted to $\mathrm{pH}$ 2. As the literature suggests the stomach $\mathrm{pH}$ of infants and young children may be higher compared with adults [32], we also conducted a subset of experiments, with a limited number of YCF, in which 
digestions were initiated with a gastric phase $\mathrm{pH}$ of 4 . During the gastric phase (at both $\mathrm{pHs}$ ), digestates were placed in a shaking incubator for $75 \mathrm{~min}$. Subsequently, the $\mathrm{pH}$ was raised to 5.0-6.0, bile salts and pancreatin digestive enzymes were added, and the $\mathrm{pH}$ was readjusted to 6.9-7.0 followed by another $75 \mathrm{~min}$ in the shaking incubator to mimic the small intestinal phase of digestion.

A chamber was created over each individual cell culture well containing the Caco-2 cell monolayer using a 15 $\mathrm{kD}$ molecular weight cut-off dialysis membrane fitted over a Transwell insert and held in place with a silicon ring. $1.5 \mathrm{~mL}$ of the digestate was then placed in the chamber that was in contact with the cell culture media of the well. The tissue culture plates were placed in a $37{ }^{\circ} \mathrm{C}$ incubator for $60 \mathrm{~min}$, while in the incubator they were rotated using a platform-fitted multi-function 3D rotator set at 6 oscillations/min. The inserts were then removed and an additional $1 \mathrm{~mL}$ of supplemented MEM added to each well. The cells were returned to the incubator for the following $22 \mathrm{~h}$ and then harvested for ferritin.

All YCF samples were tested on a minimum of three separate occasions at gastric phase $\mathrm{pH} \mathrm{2,}$ with $n=6$ for each treatment. Further in vitro digestions were carried out on one or more occasions with $n \geq 6$ (specified on figures and tables) for: gastric phase digestion at $\mathrm{pH} 4$; YCF with exogenously added GOS and FOS so that total levels of prebiotics were equivalent across all YCF samples; YCF with exogenously added ascorbic acid so that levels of ascorbic acid were equivalent across all YCF samples; YCF with exogenously added GOS, FOS and ascorbic acid so that levels were equivalent across all YCF; and heat-treated digestates.

\section{Cell harvest and ferritin analysis}

Cells were harvested as previously described [27, 28]. In brief, the monolayers of cells were rinsed and then detached with cell lysis buffer (CelLyticTM-Sigma-Aldrich) and subsequently centrifuged at $6000 \times g$ for $6 \mathrm{~min}$; supernatant was separated and stored at $-80^{\circ} \mathrm{C}$. The samples were analysed for ferritin using the SpectroFerritin MT Enzyme Linked Immunoassay (ELISA; RAMCO, TX, USA). Ferritin levels were adjusted for varying cell numbers/well by measurement of cell protein/well (Pierce Protein BCA assay) and expressed as ng ferritin/mg of cell protein.

\section{Ascorbic acid analysis}

All samples, and GOS and FOS used to fortify Samples $\mathrm{C}$ and D to levels equivalent with Samples A and B, were analysed for reduced ascorbic acid (herein referred to rAA) concentrations using the 2,6-Dichloroindophenol Titrimetric method (AOAC 985.33). Briefly, $5 \mathrm{ml}$ of the
YCF being analysed, or $5 \mathrm{~g}$ of the GOS and FOS powders being tested (prebiotic powders were initially ground in a mortar and pestle with fine sand, then strained), were added to a mixture of glacial acetic acid and metaphosphoric acid, and titrated with a freshly prepared indophenol standard solution. A rAA standard solution was freshly prepared and used to standardise the indophenol titration solution. rAA levels were calculated based on the ascorbic acid equivalent mass titrated to $1 \mathrm{ml}$ of indophenol indicator solution (in relation to the rAA standard solution), adjusted for dilutions factors and the test solution blank titration level.

\section{Heat treatment}

To evaluate the contribution of ascorbic acid to iron uptake, selected YCF digestates, and the iron plus ascorbic acid positive control, were treated for $7.5 \mathrm{~min}$ in a $100{ }^{\circ} \mathrm{C}$ water bath and then plunged into ice, in order to degrade the ascorbic acid [33].

\section{Statistical analysis}

Statistical analysis of the data was performed using GraphPad Prism (version 6.0c GraphPad Software, San Diego, CA, USA). In vitro digestion experiments were analysed using the statistical methods of Motulsky [34]. Data are presented as mean \pm SEM and were analysed by one-way ANOVA followed by either Tukey's multiple comparisons test (all-pairwise across experimental groups) or, where the comparison being made was only between select pairs, Sidak's multiple comparisons test. Differences between means were considered significant at $p \leq 0.05$. Ordered increases in data were tested using the nonparametric Jonckheere-Terpstra test. Ordered increases were considered significant at $p \leq 0.05$. The Jonckheere-Terpstra test was computed in Microsoft Excel. Unless stated otherwise, in the results section the word "significantly" is used to denote statistical significance as indicated by the relevant $p$ shown in parenthesis at the end of the statement.

\section{Results}

\section{Iron bioavailability of Tested YCF at gastric phase pH 2}

Data on iron bioavailability of YCF A-D from the in vitro digestion/Caco-2 cell model are presented in Fig. 1.

YCF A and B, which had the highest levels of prebiotics and ascorbic acid, had the highest iron bioavailability as measured by ferritin formation. YCF A ferritin levels were 43 , and $62 \%$ greater than YCF C and D, respectively $(p<0.0001)$. YCF B ferritin levels were $25 \%$, greater 
than YCF C, although this was not statistically significant ( $p=0.1007)$, and $41 \%$ greater than YCF D $(p=0.0113)$. YCF C and D did not have statistically different ferritin concentrations.

\section{Iron bioavailability of tested YCF at gastric pH 4}

YCF A, B and D (representing the YCF with the highest and lowest bioavailability, respectively) were tested using a gastric phase $\mathrm{pH}$ of 4 (Table 2). Changing the $\mathrm{pH}$ to $4 \mathrm{did}$ not alter iron bioavailability for any of the tested YCF.

\section{Iron bioavailability of YCF to which exogenous GOS and FOS were added}

Data on iron bioavailability for these experiments are presented in Fig. 1.

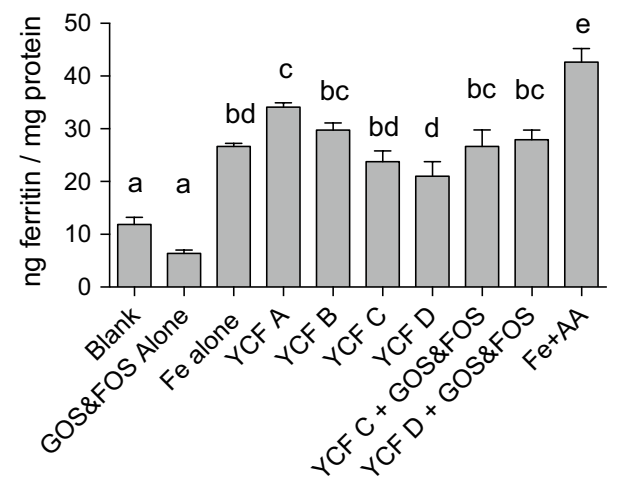

Fig. 1 Caco-2 ferritin levels from treatment of cells with digestates of controls, YCF A-D, and YCF C and D supplemented with GOS and FOS to levels equivalent to those found in YCF A and B. Values presented are means of data normalised to the Fe reference control (Fe alone) \pm SEM ( $n \geq 18$ for YCF alone, all others $n \geq 6$ ). Treatment with YCF A yielded the highest ferritin compared with all other YCF although not statistical when compared to YCF B. The addition of GOS and FOS to YCF C and D increased ferritin to levels statistically equivalent to YCF A and B. Based on an ANOVA $(p<0.0001)$ with Tukey's multiple comparisons test, post hoc analysis done on an all-pairwise basis; means with different superscript letters in a column are statistically different $(p \leq 0.05)$

Table 2 Ferritin values of Caco-2 cells treated with YCF A, B and D at gastric phase digestions of $\mathrm{pH} 2$ compared with $\mathrm{pH} 4$

\begin{tabular}{llll}
\hline YCF sample & $\begin{array}{l}\mathrm{pH} 2 \\
\text { ng ferritin/mg protein }\end{array}$ & $\begin{array}{l}\mathrm{pH} 4 \\
\text { ng ferritin/mg protein }\end{array}$ & $p$ value \\
\hline YCF A & $34.1 \pm 0.9$ & $29.9 \pm 1.1$ & 0.4239 \\
YCF B & $29.7 \pm 1.4$ & $30.1 \pm 2.5$ & 0.9990 \\
YCF D & $21.1 \pm 2.8$ & $17.1 \pm 3.2$ & 0.5407 \\
\hline
\end{tabular}

Values presented are the mean \pm SEM. There was no statistical difference between $\mathrm{pH} 2$ and $\mathrm{pH} 4$ gastric phase digestions for any of the three samples using Sidak's multiple comparisons test $(n \geq 6)$
Ferritin levels in YCF C and D were significantly lower than those in YCF A. Ferritin levels of YCF C were $10.25 \mathrm{ng}$ ferritin/mg protein lower than YCF A $(30 \%$ lower; $p<0.0001$ ), and ferritin levels of YCF D were $13.01 \mathrm{ng}$ ferritin/mg protein lower than YCF A (38\% lower; $p<0.0001)$. While not statistically significant, ferritin levels of YCF C were also 20\% lower than YCF B ferritin levels $(p=0.1007)$. YCF D was significantly lower than YCF B by $29 \%$ ( $p=0.0113)$. YCF C originally contained $0.5 \backslash, \mathrm{g}$ GOS/100 ml milk, whereas YCF D originally contained no supplemental prebiotics. After the addition of a mixture of GOS and FOS to both YCF C and D, so that total prebiotic levels were equivalent to those found in YCF A and B, there
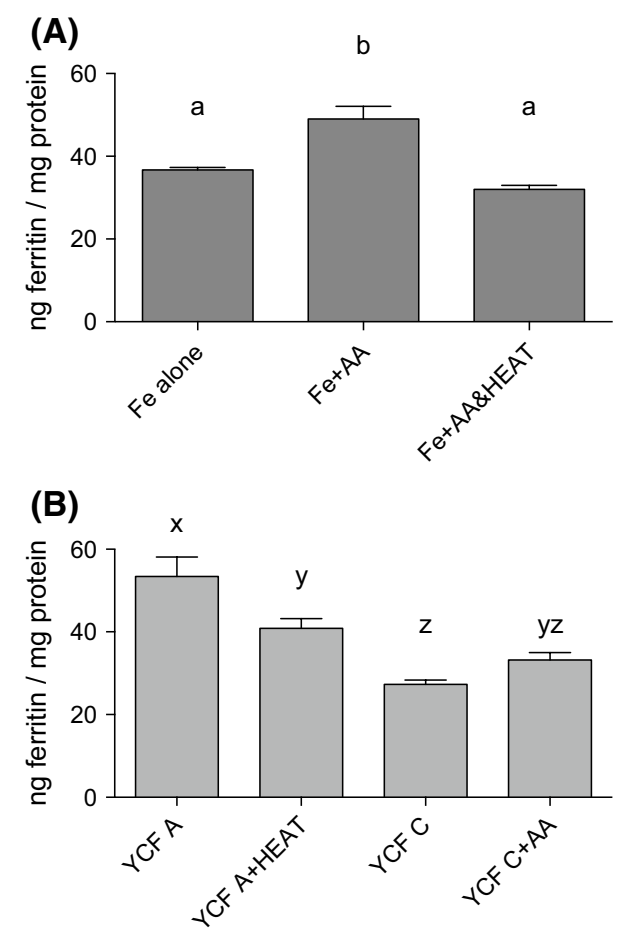

Fig. 2 a Ferritin levels of Caco-2 cells exposed to control digests, both with, and without, heat treatment to degrade ascorbic acid (AA). Heat treatment of the positive control $(\mathrm{Fe}+\mathrm{AA}$ at a 1:10 Fe/AA molar ratio) decreased the enhancing effect of AA on iron uptake; ferritin formation was equivalent between the $\mathrm{Fe}$ alone and $\mathrm{Fe}+\mathrm{AA}$ controls. Based on an ANOVA ( $p=0.0003$ ) with Tukey's multiple comparison test, post hoc analysis done on an all-pairwise basis; means with different superscript letters in a column are statistically different ( $p \leq 0.0015)$. b Caco-2 ferritin levels of cells treated with YCF A digestates, with, and without, heat treatment, and YCF C digestates, with, and without, added $\mathrm{AA}(\approx 1: 4 \mathrm{Fe} / \mathrm{AA}$ molar ratio). Ferritin levels of YCF A decreased after heat treatment; ferritin levels of YCF C increased after the addition of AA to levels equivalent with YCF A; however, ferritin was still less than non-heat-treated YCF A levels. All presented values are means normalised to the Fe reference control (Fe alone) \pm SEM. Based on an ANOVA $(p<0.0001)$ with Tukey's multiple comparisons test, post hoc analysis done on an allpairwise basis; means with different superscript letters in a column are statistically different $(p \leq 0.0074)$ 
was no longer a statistically significant difference between the ferritin levels in any of the four tested YCF although YCF C and D ferritin levels were still 7.42 and 6.15 ng ferritin/mg protein lower (by 22 and 18\%), respectively, compared with YCF A.

\section{Iron bioavailability of YCF relative to ascorbic acid with and without heat treatment}

YCF A and B had equal amounts of ascorbic acid according to stated information on the manufacturer's label (15 mg ascorbic acid/100 ml milk); measured levels were 14.2 and $16.3 \mathrm{mg} \mathrm{rAA} / 100 \mathrm{ml}$ milk, respectively. Ascorbic acid levels in YCF C were reported as $10 \mathrm{mg} / 100 \mathrm{ml}$ milk (the lowest of all tested YCF) and measured as $10.2 \mathrm{mg} / 100 \mathrm{ml}$; YCF D rAA levels were reported as $12 \mathrm{mg} / 100 \mathrm{ml}$ and measured as $10.1 \mathrm{mg} / 100 \mathrm{ml}$.

The reported ascorbic acid/iron molar ratios were 4:1 for YCF A and B and 3:1 for YCF C and D, respectively. Ascorbic acid/iron molar ratios based on measured rAA were: $3.75: 1 ; 4.3: 1 ; 3: 1$; and 2.7:1; for YCF A, B, C and D, respectively.

In order to ensure that our heat treatment inactivated the ascorbic acid present in YCF samples, we tested whether heat-treating our positive control (Fe + AA) affected ferritin formation. Heat treatment of the positive control significantly decreased ferritin levels compared with the non-heat-treated positive control $(p=0.0002)$; levels were equivalent to those seen in our iron alone controls (i.e. iron added to digestates without any added ascorbic acid; $p=0.2227$ ) (Fig. 2a). Heat treatment of YCF A significantly decreased ferritin levels by approximately $24 \%$ (from 53.42 to $40.83 \mathrm{ng}$ ferritin/mg protein; $p=0.0074$ ); however, YCF A ferritin concentration, even after heat treatment, was still significantly higher by $13.52 \mathrm{ng}$ ferritin/ mg protein compared with YCF C levels (the sample with the lowest reported and measured AA levels; $p=0.0082$ ). Lastly, we added exogenous ascorbic acid to YCF $\mathrm{C}$ to levels equivalent to those found in YCF A; ferritin levels increased by $5.90 \mathrm{ng}$ ferritin/mg protein, or approximately $22 \%$, but were still $20.20 \mathrm{ng}$ ferritin/mg protein less (38\%) than those measured in cells treated with YCF A $(p=0.0002$; Fig. $2 b)$.

\section{Iron bioavailability of YCF C and D with the addition of exogenous GOS and FOS, with, or without, added ascorbic acid}

In order to test whether prebiotics and ascorbic acid had an additive effect on iron bioavailability, we measured ferritin levels of digestates of YCF $\mathrm{C}$ and D with added GOS and FOS alone, compared with digestates of YCF $\mathrm{C}$ and $\mathrm{D}$ with added GOS

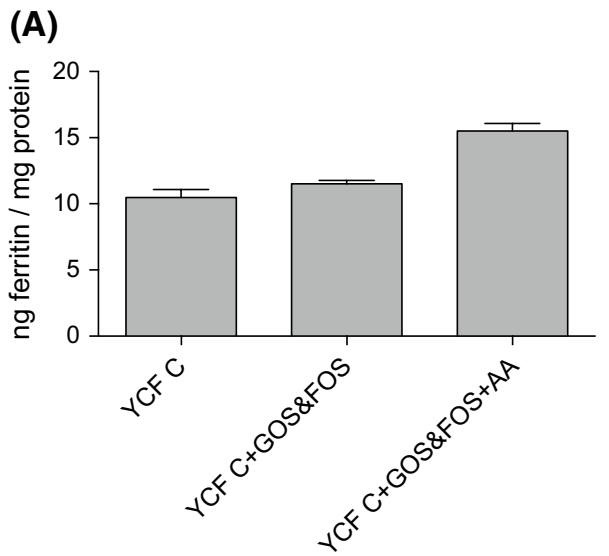

(B)

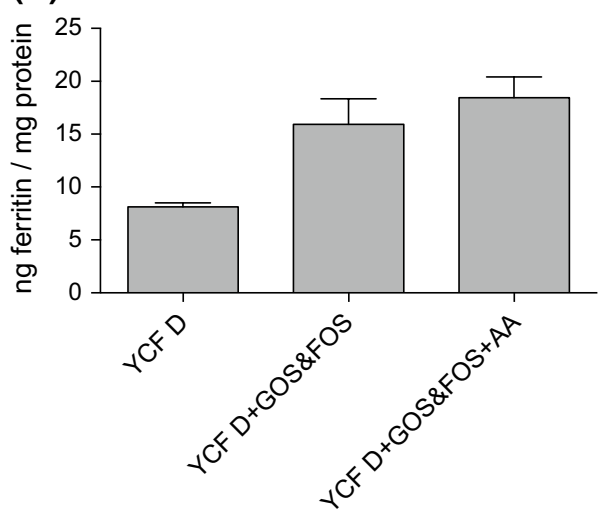

Fig. 3 a Ferritin levels of Caco-2 cells treated with digests of: YCF C alone; YCF C + GOS and FOS; and YCF C, GOS and FOS, +ascorbic acid $(\mathrm{AA}, 114 \mu \mathrm{mol} / \mathrm{L} ; \approx 1: 4 \mathrm{Fe} / \mathrm{AA}$ molar ratio). Increase in ferritin formation was tested using the Jonckheere-Terpstra test and is significant in the order YCF C alone, YCF C + GOS and FOS, and YCF C, GOS and FOS, +AA $(p=0.0048)$. Ferritin levels of Caco-2 cells treated with digests of: YCF D alone; YCF D + GOS and FOS; and YCF D, GOS and FOS, + AA $(114 \mu \mathrm{mol} / \mathrm{L} ; \approx 1: 4$ $\mathrm{Fe} / \mathrm{AA}$ molar ratio). Increase in ferritin formation was tested using the Jonckheere-Terpstra test and is significant in the order YCF D alone, YCF D + GOS and FOS, and YCF D, GOS and FOS, and AA $(p=0.0001)$

and FOS and ascorbic acid. There was an ordered increase in ferritin formation in the order of digestates of YCF C alone, YCF C and GOS and FOS, and YCF C, GOS and FOS and ascorbic acid ( $p=0.0048$; Fig. 3a); similarly, there was an ordered increase in ferritin formation in the order of digestates of YCF D alone, YCF D and GOS and FOS, and YCF D, GOS and FOS and ascorbic acid $(p=0.0001 ;$ Fig. 3b).

\section{Discussion}

YCF A and B, with the highest levels of prebiotics and ascorbic acid, had the highest iron bioavailability as measured by Caco- 2 cell ferritin formation. YCF A ferritin levels 
were approximately 1.5 times greater than those of YCF C and D, while YCF B ferritin levels were approximately 1.3 times greater than those of YCF C and D. The addition of prebiotics (a mixture of GOS and FOS) and ascorbic acid to YCF C and D increased iron uptake by approximately 1.5 and 2.3 times, respectively, but neither exogenously added ascorbic acid alone, nor prebiotics alone, elevated ferritin levels to those seen in YCF A, suggesting both factors contributed to the greater iron bioavailability of YCF A.

Previous reports in the literature found that an ascorbic acid to iron molar ration of $4: 1$ is associated with higher iron bioavailability in high-phytate food products [35]; consistent with this in our study YCF A and B, which had the most bioavailable iron, also had both higher absolute amounts of ascorbic acid and higher ascorbic acid to iron molar ratios compared with the other tested YCF.

Relative to prebiotics, the results of our study are consistent with numerous studies, demonstrating prebiotics enhances iron absorption in rodents and pigs [36-40]. Potential mechanisms driving this increase included: enhanced solubility of iron [40]; a decrease in colonic $\mathrm{pH}[41,42]$; changes in the profile of the gut microbiota [43]; a direct effect on iron transporter expression in either duodenum or colon $[36,44,45]$; or a systemic decrease in inflammation leading to decreased production of hepcidin, a peptide secreted by the liver in response to inflammation that decreases iron absorption [41]. However, it is not clear whether any of these mechanisms would be relevant in our short-term in vitro studies.

Results from studies evaluating the effects of prebiotics on iron availability carried out in human beings, and also in vitro with the Caco- 2 cell line, have been mixed. A recent study in women with low iron status who were given approximately $20 \mathrm{~g} / \mathrm{d}$ of the FOS-inulin found that although inulin did modify the large bowel microbiome and also lowered colonic $\mathrm{pH}$, there was no influence on iron absorption as measured by the stable isotope technique; iron absorption in the inulin group was $14 \%$ higher, but this was not statistically significant [46]. Another study carried out in young men using the same method to assess iron absorption also found no statistically significant effect of inulin, FOS, or GOS on iron absorption, though absorption was some $20 \%$ higher in the FOS group compared with control [47]. Both of these studies were relatively small ( $n=32, n=15$, respectively) and therefore may have been underpowered to detect small, but physiologically relevant, increases in iron uptake. Absorption of iron is primarily determined by host iron status, and differences in iron absorption from foods containing less potent enhancers of absorption (e.g. prebiotics) may not be revealed unless some degree of iron deficiency is present, or absolute amounts of bioavailable dietary iron are too low to meet needs. Our results are consistent with a study carried out in infants and young children that demonstrated improved iron status amongst children receiving a mixture of prebiotic oligosaccharides (type not specified) and probiotics [48]. A possible reason for these different study results is that inulin may not be the optimal FOS for increasing iron absorption. Shorter-chain FOS more closely resemble fructose [49], which we have shown increases non-haem iron bioavailability in the Caco-2 cell model [27]. Our previous work demonstrated that fructose added to a ferric iron solution increased levels of ferrous iron by approximately $300 \%$ [27], suggesting that the observed increase in iron bioavailability was caused by fructose reducing ferric iron to the more bioavailable ferrous iron. Shorter-chain FOS may be better at improving iron bioavailability compared with longer-chain FOS-inulin if they share some degree of this reducing effect, or secondary to other structural-related differences. In support of this, recent work using GOS suggests that specific structural differences in prebiotics alter their influence on iron absorption [50]. Lastly, human studies evaluating the effects of GOS and FOS given together on iron bioavailability have not, to the authors' knowledge, been conducted; the two prebiotics together may act synergistically.

Several in vitro studies evaluating the effect of prebiotics on iron uptake have been carried out in the past decade. Two Caco-2 cell-based studies evaluating iron bioavailability from milk- or soy-based yogurts, and common beans, both found that inulin did not improve iron bioavailability from the test food matrix [25, 42]. In contrast, and in agreement with our findings, another in vitro study using ironfortified cereal biscuits, to which inulin had been added, demonstrated improved iron bioavailability that correlated with increased iron solubility [51]. Both the yogurt and bean-based studies used a higher dose of inulin compared with our study (approximately $17 \mathrm{mg}$ vs. $2.4 \mathrm{mg}$ per digestate) and did not add any GOS; the amount of prebiotics applied to Caco-2 cells in the biscuit study was not specified. In conclusion, the positive results in our present study may relate to several factors: different dose of FOS; different forms of FOS and GOS; and the addition of GOS and FOS together to the tested food matrix.

Young children have the highest risk of ID/IDA amongst pre-adolescent children. The ESPGHAN Committee on Nutrition recommends young children receive a diet rich with iron-containing foods and that excess intake of ironpoor cow's milk be avoided [3]. However, ID/IDA persists in young children, and thus YCF fortified with iron may be beneficial, as part of a varied diet, in the prevention of ID/ IDA. In addition, fortified milk products with the most bioavailable iron would also be predicted to deliver less unabsorbed iron to the young colon. The findings of this study suggest that both prebiotics and ascorbic acid contribute to improved iron bioavailability in YCF. However, ascorbic 
acid is a relatively unstable micronutrient, and levels may fluctuate depending on storage conditions and timing of use from purchase date [33], whereas prebiotics are relatively stable in foods $[52,53]$. Thus, the use of both dietary factors to improve iron bioavailability in fortified milks may be complementary.

Several observational studies in Europe [10, 54] found that intake of YCF was associated with increased dietary intake of iron in young children. A recent cross-sectional study carried out in Western Europe amongst healthy young children found that 11.9 and $3.9 \%$ of tested children had ID, and IDA, respectively, and use of cow's milk as the main milk source was associated with an increased risk of ID [55]. The prevalence of ID in children primarily receiving cow's milk was $19.7 \%$ in contrast with children whose main source of milk was YCF (ID prevalence 5.4\%); this difference was highly significant $(p<0.001)$ [55]. The specific YCF used in this study were not specified, nor were study participants' intakes of ascorbic acid or prebiotics.

There are several limitations to our study. Although the Caco-2 cell system is a validated model for assessing iron bioavailability including in the food matrixes we tested, the magnitude of effects may not always be accurate [56]. It thus remains for our results to be confirmed in vivo. In addition, we did not analyse the specific forms of GOS and FOS used in this study, and there is evidence that there is structural specificity in terms of the effects of prebiotics on iron bioavailability. Studies looking at specific prebiotic structure and its relation to iron availability would be helpful. Furthermore, although the European Commission regulates the absolute amounts both of prebiotics (and the ratio of GOS to FOS) that may be added to YCF, dose-response analysis of prebiotic effects on iron bioavailability would enable producers to optimise formulation.

In conclusion, use of YCF may contribute significantly to iron intake in young children and thus potentially reduce ID/IDA; however, in the case of iron, the absolute amount ingested does not necessarily reflect the fraction absorbed. Therefore, ensuring that iron in YCF is delivered in a bioavailable form, while minimising the delivery of excess iron to the young gut, would improve the nutritional benefits of YCF. Our results suggest that ascorbic acid and prebiotics in YCF improve iron bioavailability. Further in vivo work to confirm our results and also to explore whether absolute amounts of iron fortification in YCF could be decreased through optimisation of concentration and forms of prebiotics and ascorbic acid warrants investigation.

Acknowledgements The Faculty of Engineering and Science at the University of Greenwich, and the Diabetes and Nutritional Sciences Division at King's College London, supported this work. We thank David Scott Ganis for assistance with statistical and numerical analysis.

\section{Compliance with ethical standards}

Conflict of interest All authors declare that they have no conflict of interest.

Open Access This article is distributed under the terms of the Creative Commons Attribution 4.0 International License (http://creativecommons.org/licenses/by/4.0/), which permits unrestricted use, distribution, and reproduction in any medium, provided you give appropriate credit to the original author(s) and the source, provide a link to the Creative Commons license, and indicate if changes were made.

\section{References}

1. Nyaradi A, Li J, Hickling S, Foster J, Oddy WH (2013) The role of nutrition in children's neurocognitive development, from pregnancy through childhood. Front Hum Neurosci 7:97

2. Lozoff B, Jimenez E, Hagen J, Mollen E, Wolf AW (2000) Poorer behavioral and developmental outcome more than 10 years after treatment for iron deficiency in infancy. Pediatrics 105:E51

3. Domellof M, Braegger C, Campoy C, Colomb V, Decsi T, Fewtrell $\mathrm{M}$ et al (2014) Iron requirements of infants and toddlers. J Pediatr Gastroenterol Nutr 58:119-129

4. Paoletti G, Bogen DL, Ritchey AK (2014) Severe iron-deficiency anemia still an issue in toddlers. Clin Pediatr (Phila) 53:1352-1358

5. American Academy of Pediatrics Committee on Nutrition (1989) Iron-fortified infant formulas. Pediatrics 84:1114-1115

6. Scientific Advisory Committee on Nutrition (SACN) (2010) Iron and health. Department of Health, TSO, London

7. Brotanek JM, Gosz J, Weitzman M, Flores G (2008) Secular trends in the prevalence of iron deficiency among us toddlers, 1976-2002. Arch Pediatr Adolesc Med 162:374-381

8. Bates B, Lennox A, Prentice A, Bates C, Page P, Nicholson S, Swan G (2014) National diet and nutrition survey: results from years 1-4 (combined) of the rolling programme (2008/20092011/12): Executive summary. Department of Health, TSO, London

9. Ziegler EE (2011) Consumption of cow's milk as a cause of iron deficiency in infants and toddlers. Nutr Rev 69(Suppl 1):S37-S42

10. Ghisolfi J, Fantino M, Turck D, de Courcy GP, Vidailhet M (2013) Nutrient intakes of children aged 1-2 years as a function of milk consumption, cows' milk or growing-up milk. Public Health Nutr 16:524-534

11. McCarthy EK, Ni Chaoimh C, O'B Hourihane J, Kenny LC, Irvine AD, Murray DM, Kiely M (2016) Iron intakes and status of 2-year-old children in the Cork BASELINE Birth Cohort Study. Matern Child Nutr. doi:10.1111/mcn.12320

12. Lennox ASJ, Ong K, Henderson H, Allen R (2011) Diet and nutrition survey of infants and young children: executive summary. Department of Health, TSO, London

13. Gibson GR, Roberfroid MB (1995) Dietary modulation of the human colonic microbiota: introducing the concept of prebiotics. J Nutr 125:1401-1412

14. Gibson GR, Probert HM, Loo JV, Rastall RA, Roberfroid MB (2004) Dietary modulation of the human colonic microbiota: updating the concept of prebiotics. Nutr Res Rev 17:259-275

15. Yeung C, Glahn RP, Welch RM, Miller DD (2005) Prebiotics and iron bioavailability-is there a connection? J Food Sci 70:88-92 
16. Braegger C, Chmielewska A, Decsi T, Kolacek S, Mihatsch W, Moreno L et al (2011) Supplementation of infant formula with probiotics and/or prebiotics: a systematic review and comment by the ESPGHAN Committee on Nutrition. J Pediatr Gastroenterol Nutr 52:238-250

17. Commission Directive 2006/141/EC of 22 December 2006 on infant formulae and follow-on formulae and amending Directive 1999/21/EC (Text with EEA relevance). Official Journal of the European Union. 30 December 2006. L401/1. http://eur-lex. europa.eu/LexUriServ/LexUriServ.do?uri=OJ:L:2006:401:0001 :0033:EN:PDF. 11 Sept 2016

18. Krebs NF, Domellof M, Ziegler E (2015) Balancing benefits and risks of iron fortification in resource-rich countries. J Pediatr 167:S20-S25

19. Jaeggi T, Kortman GA, Moretti D, Chassard C, Holding P, Dostal A et al (2015) Iron fortification adversely affects the gut microbiome, increases pathogen abundance and induces intestinal inflammation in kenyan infants. Gut 64:731-742

20. Zimmermann MB, Chassard C, Rohner F, N'Goran EK, Nindjin C, Dostal A et al (2010) The effects of iron fortification on the gut microbiota in African children: a randomized controlled trial in Cote d'Ivoire. Am J Clin Nutr 92:1406-1415

21. Quinn EA (2014) Too much of a good thing: evolutionary perspectives on infant formula fortification in the United States and its effects on infant health. Am J Hum Biol 26:10-17

22. Singhal A, Morley R, Abbott R, Fairweather-Tait S, Stephenson T, Lucas A (2000) Clinical safety of iron-fortified formulas. Pediatrics 105:E38

23. Etcheverry P, Wallingford JC, Miller DD, Glahn RP (2004) Calcium, zinc, and iron bioavailabilities from a commercial human milk fortifier: a comparison study. J Dairy Sci 87:3629-3637

24. Viadel B, Perales S, Barbera R, Lagarda M, Farre R (2007) Ferritin synthesis by Caco-2 cells as an indicator of iron bioavailability: application to milk-based infant formulas. Food Chem 102:925-931

25. Laparra JM, Tako E, Glahn RP, Miller DD (2008) Supplemental inulin does not enhance iron bioavailability to Caco- 2 cells from milk- or soy-based, probiotic-containing, yogurts but incubation at 37 degrees c does. Food Chem 109:122-128

26. Caro IBX, Rousset M, Meunier V, Bourrie M, Julian B, Joyeux H, Roques C, Berger Y, Zweibaum A, Fabre G (1995) Characterisation of a newly isolated Caco-2 clone (TC7), as a model of transport processes and biotransformation of drugs. Int J Pharm 116:147-158

27. Christides T, Sharp P (2013) Sugars increase non-heme iron bioavailability in human epithelial intestinal and liver cells. PLoS ONE 8:e83031

28. Christides T, Wray D, McBride R, Fairweather R, Sharp P (2014) Iron bioavailability from commercially available iron supplements. Eur J Nutr. doi:10.1007/s00394-014-0815-8

29. Johnson DM, Yamaji S, Tennant J, Srai SK, Sharp PA (2005) Regulation of divalent metal transporter expression in human intestinal epithelial cells following exposure to non-haem iron. FEBS Lett 579:1923-1929

30. Glahn RP, Lee OA, Yeung A, Goldman MI, Miller DD (1998) Caco-2 cell ferritin formation predicts nonradiolabeled food iron availability in an in vitro digestion/Caco-2 cell culture model. J Nutr 128:1555-1561

31. Jumarie C, Malo C (1991) Caco-2 cells cultured in serum-free medium as a model for the study of enterocytic differentiation in vitro. J Cell Physiol 149:24-33

32. Bowles A, Keane J, Ernest T, Clapham D, Tuleu C (2010) Specific aspects of gastro-intestinal transit in children for drug delivery design. Int J Pharm 395:37-43

33. Davey MWMM, Inzé D, Sanmartin M, Kanellis A, Smirnoff N, Benzie IJ, Strain JJ, Favell D, Fletcher J (2000) Plant L-ascorbic acid: chemistry, function, metabolism, bioavailability and effects of processing. J Sci Food Agric 80:825-860

34. Motulsky H (2010) Intuitive biostatistics, 2nd edn. Oxford University Press, Oxford

35. Allen L, de Benoist B, Dary O, Hurrell R (2006) In: Guidelines on food fortification with micronutrients. World Health Organization, Dept. of Nutrition for Health and Development

36. Lobo AR, Gaievski EH, De Carli E, Alvares EP, Colli C (2014) Fructo-oligosaccharides and iron bioavailability in anaemic rats: the effects on iron species distribution, ferroportin-1 expression, crypt bifurcation and crypt cell proliferation in the caecum. Br J Nutr 112:1286-1295

37. Freitas Kde C, Amancio OM, de Morais MB (2012) High-performance inulin and oligofructose prebiotics increase the intestinal absorption of iron in rats with iron deficiency anaemia during the growth phase. Br J Nutr 108:1008-1016

38. Wang Y, Zeng T, Wang SE, Wang W, Wang Q, Yu HX (2010) Fructo-oligosaccharides enhance the mineral absorption and counteract the adverse effects of phytic acid in mice. Nutrition 26:305-311

39. Pérez-Conesa D, López G, Ros G (2007) Effect of probiotic, prebiotic and synbiotic follow-up infant formulas on iron bioavailability in rats. Food Sci Technol Int 13:69-77

40. Yasuda K, Roneker KR, Miller DD, Welch RM, Lei XG (2006) Supplemental dietary inulin affects the bioavailability of iron in corn and soybean meal to young pigs. J Nutr 136:3033-3038

41. Yasuda K, Dawson HD, Wasmuth EV, Roneker CA, Chen C, Urban JF et al (2009) Supplemental dietary inulin influences expression of iron and inflammation related genes in young pigs. J Nutr 139:2018-2023

42. Laparra JM, Glahn RP, Miller DD (2009) Assessing potential effects of inulin and probiotic bacteria on $\mathrm{Fe}$ availability from common beans (Phaseolus vulgaris L.) to Caco-2 cells. J Food Sci 74:H40-H46

43. Patterson JK, Yasuda K, Welch RM, Miller DD, Lei XG (2010) Supplemental dietary inulin of variable chain lengths alters intestinal bacterial populations in young pigs. J Nutr 140:2158-2161

44. Tako E, Glahn RP, Welch RM, Lei X, Yasuda K, Miller DD (2008) Dietary inulin affects the expression of intestinal enterocyte iron transporters, receptors and storage protein and alters the microbiota in the pig intestine. Br J Nutr 99:472-480

45. Marciano R, Santamarina AB, de Santana AA, Silva Mde L, Amancio OM, do Nascimento CM et al (2015) Effects of prebiotic supplementation on the expression of proteins regulating iron absorption in anaemic growing rats. Br J Nutr 113:901-908

46. Petry N, Egli I, Chassard C, Lacroix C, Hurrell R (2012) Inulin modifies the Bifidobacteria population, fecal lactate concentration, and fecal $\mathrm{pH}$ but does not influence iron absorption in women with low iron status. Am J Clin Nutr 96:325-331

47. van den Heuvel EG, Schaafsma G, Muys T, van Dokkum W (1998) Nondigestible oligosaccharides do not interfere with calcium and nonheme-iron absorption in young, healthy men. Am J Clin Nutr 67:445-451

48. Sazawal S, Dhingra U, Hiremath G, Sarkar A, Dhingra P, Dutta A et al (2010) Effects of Bifidobacterium lactis hn019 and prebiotic oligosaccharide added to milk on iron status, anemia, and growth among children 1 to 4 years old. J Pediatr Gastroenterol Nutr 51:341-346

49. Schaafsma G, Slavin JL (2015) Significance of inulin fructans in the human diet. Compr Rev Food Sci Food Saf 14:37-47

50. Laparra JM, Diez-Municio M, Herrero M, Moreno FJ (2014) Structural differences of prebiotic oligosaccharides influence their capability to enhance iron absorption in deficient rats. Food Funct 5:2430-2437 
51. Vitali DRM, Cetina-Čižmek B, Dragojević Vedrina (2011) Caco-2 cell uptake of $\mathrm{Ca}, \mathrm{Mg}$ and $\mathrm{Fe}$ from biscuits as affected by enrichment with pseudocereal/inulin mixtures. Acta Aliment 40:480-489

52. Klewicki R (2007) The stability of gal-polyols and oligosaccharides during pasteurization at a low $\mathrm{pH}$. Food Sci Technol-LEB 40:1259-1265

53. Vega R, Zuniga-Hansen ME (2015) The effect of processing conditions on the stability of fructooligosaccharides in acidic food products. Food Chem 173:784-789

54. Walton J, Flynn A (2013) Nutritional adequacy of diets containing growing up milks or unfortified cow's milk in irish children (aged 12-24 months). Food Nutr Res. doi:10.3402/fnr. v57i0.21836
55. Akkermans MD, van der Horst-Graat JM, Eussen SR, van Goudoever JB, Brus F (2016) Iron and vitamin D deficiency in healthy young children in Western Europe despite current nutritional recommendations. J Pediatr Gastroenterol Nutr 62:635-642

56. Fairweather-Tait S, Lynch S, Hotz C, Hurrell R, Abrahamse L, Beebe $S$ et al (2005) The usefulness of in vitro models to predict the bioavailability of iron and zinc: a consensus statement from the harvestplus expert consultation. Int J Vitam Nutr Res 75:371-374 